Walter Dambrosio · Duccio Papini

\title{
Periodic solutions of asymptotically linear second order equations with indefinite weight ${ }^{\star}$
}

Received: January 9, 2003; in final form: September 20, 2003

Published online: July 2, 2004 - (c) Springer-Verlag 2004

Abstract. In this paper we study the ordinary differential equation

$$
\ddot{x}+q(t) g(x)=0,
$$

where $g$ is a locally Lipschitz continuous function that satisfies $g(x) x>0$ for all $x \neq 0$ and is asymptotically linear, while $q$ is a continuous, $\pi$-periodic and changing sign weight. By the application of a recent result on the existence and multiplicity of fixed points of planar maps, we give conditions on $q$ and on the behavior of the ratio $g(x) / x$ near zero and near infinity in order to obtain multiple periodic solutions with the prescribed number of zeros in the intervals of positivity and negativity of $q$, as well as multiple subharmonics of any order and uncountably many bounded solutions.

Mathematics Subject Classification (2000). 34B09, 34C25, 34C28

\section{Introduction}

In this paper we study the periodic problem associated to an equation of the form

$$
\ddot{x}+q(t) g(x)=0,
$$

where $g \in C(\mathbf{R})$ satisfies $0<g(x) / x<M$, for all $x \neq 0$ and for some $M>0$, and $q \in C(\mathbf{R})$ is $\pi$-periodic and assumes both positive and negative values.

In the literature, differential equations of this kind and their generalization in higher dimension are often called "indefinite in sign". In the last years many boundary value problems associated to this type of equation have been studied. The results obtained are mostly valid for nonlinearities that either have superlinear growth at infinity or are sublinear near the origin or at infinity. We quote for instance the papers $[1-4,6,8,9,11,12,14,16,18]$ and the bibliography contained therein.

W. Dambrosio: Dipartimento di Matematica, Università di Torino, Via Carlo Alberto 10, 10123 Torino, Italy, e-mail: dambrosio@dm. unito. it

D. Papini: Dipartimento dell'Ingegneria dell'Informazione, Università di Siena, via Roma 56, 53100 Siena, Italy, e-mail: papini@dii.unisi.it

* Under the auspices of GNAMPA-I.N.d.A.M., Italy. The work has been performed in the frame of the EEC project "Nonlinear boundary value problems: existence, multiplicity and stability of solutions", grant CHRX-CT94-0555. 
In particular, the papers $[5,15]$, on one hand, and [19], on the other, consider superlinear equations like (1.1) from the point of view of the periodic problem and of the detection of chaotic behavior. The first two papers use an approach based on the study of the Poincaré map associated to (1.1), while the last one exploits a variational method inspired by Nehari's method. However, in both cases periodic solutions with prescribed nodal behavior are found as well as infinitely many subharmonics of any order and uncountably many bounded non-periodic solutions.

In a very recent paper [17] an abstract fixed point theorem (stated here for quick reference in Section 3 as Theorem 3.1) was proved and successfully applied to (1.1) when $g$ is superlinear at infinity, recovering, as a byproduct, the situation outlined in the three papers above. More precisely Theorem 3.1 gives conditions under which a planar map $\psi$ and all its iterates have multiple fixed points and, moreover, produce a discrete dynamical system that is chaotic in the sense of "coin tossing". The main tool to reach such a goal is a detailed knowledge of which way the map $\psi$ stretches and twists paths that cross its domain.

Indeed, in the superlinear case the differential equation (1.1) has two crucial features: in any interval of positivity for $q$, the larger is the solution (in $C^{1}$ norm) the more it oscillates (twisting effect), while in any interval of negativity there are solutions that blow-up monotonically (stretching effect). These properties, which were already used by Butler in [3] to obtain infinitely many periodic solutions of (1.1), are widely exploited in [17] in order to show that the Poincaré map of the first order system associated to (1.1):

$$
\left\{\begin{array}{l}
\dot{x}=y \\
\dot{y}=-q(t) g(x)
\end{array}\right.
$$

satisfies the requirements of Theorem 3.1. As a consequence it is possible to find periodic solutions of (1.1) that have an arbitrary (and sufficiently large) number of zeros in each interval of positivity of $q$ and either none or exactly one zero in each interval of negativity, a fact that turns out to be useful when one looks for subharmonic solutions of order $m$. Moreover Theorem 3.1 provides the existence of uncountably many bounded non-periodic solutions by using the fact that in the superlinear case a solution $x$ of (1.1) is bounded in $C^{1}$ norm if and only if the numbers of zeros of $x$ inside each period of $q$ form a bounded sequence. We also notice that in proving these result the fact that $q$ changes sign plays a crucial role.

In this paper we apply Theorem 3.1 to equation (1.1) when the nonlinearity $g$ grows at most linearly. We can therefore exclude any blow-up phenomenon (all the solutions are globally continuable) and the number of oscillations of every solution of (1.1) in an interval of positivity of $q$ is bounded by $\sqrt{M}+1$, where $M$ bounds the ratio $g(x) / x$ from above.

Nevertheless, using again the abstract result in [17], it is still possible to prove the existence of a certain number of periodic solutions to (1.1). Indeed, in our main results we give conditions on $q$ and on the gap between the asymptotic behavior of the ratio $g(x) / x$, for $|x| \rightarrow 0$ and $|x| \rightarrow+\infty$, which guarantee the existence 
of multiple periodic and subharmonic solutions of (1.1) with a prescribed number of zeros in the intervals of positivity and negativity of $q$ and, again, uncountably many bounded non-periodic solutions.

The statement of the results is quite long; in this introduction we prefer to illustrate a concrete example in which the equation

$$
\ddot{x}+\left((\sin (2 t))^{+}-\mu(\sin (2 t))^{-}\right)(x+\lambda \arctan x)=0,
$$

$\lambda, \mu>0$, is considered. We observe that in (1.2) we have $q(t)=(\sin (2 t))^{+}-$ $\mu(\sin (2 t))^{-}$. As a consequence of our results, we have the following:

Corollary 1. There exists $n^{*} \in \mathbf{N}$ such that the following statement holds true: For every $N \in \mathbf{N}$ there is $\lambda_{N}>1$ such that for every $\lambda \geq \lambda_{N}$ there exists $\mu_{\lambda}>0$ such that for every $\mu \geq \mu_{\lambda}$ we have the following:

(1) for all $m \in \mathbf{N}$, for all $\delta=\left(\delta_{1}, \ldots, \delta_{m}\right) \in\{0,1\}^{m}$ and for all $\left(k_{1}, \ldots, k_{m}\right) \in$ $\{1, \ldots, N\}$ such that $m n^{*}+k_{1}+\cdots+k_{m}+\delta_{1}+\cdots+\delta_{m}$ is even, there are two $m \pi$-periodic solutions of equation (1.2), which have exactly $n^{*}+k_{j}$ zeros in $((j-1) \pi,(j-1) \pi+\pi / 2)$ and $\delta_{j}$ zeros in $((j-1) \pi+\pi / 2, j \pi)$, for every $j=1, \ldots, m$;

(2) for any pair of sequences $\delta=\left(\ldots, \delta_{-1}, \delta_{0}, \delta_{1}, \ldots\right) \in\{0,1\}^{\mathbf{Z}}$ and $k=$ $\left(\ldots, k_{-1}, k_{0}, k_{1}, \ldots\right) \in\{1, \ldots, N\}^{\mathbf{Z}}$ there are two bounded solutions of (1.2) which have exactly $n^{*}+k_{j}$ zeros in $((j-1) \pi,(j-1) \pi+\pi / 2)$ and $\delta_{j}$ zeros in $((j-1) \pi+\pi / 2, j \pi)$, for every $j \in \mathbf{Z}$

( $n^{*}$ is a natural number that does not depend on $N, \lambda$, and $\mu$ ).

Roughly speaking, the assumption on $\lambda$ in Corollary 1 guarantees that in the interval of positivity of the weight function $q$ the number of oscillations of the solutions is large (but still bounded). On the other hand, when $\mu$ is large we can ensure that, in the interval of negativity of $q$, certain solutions having small initial values become large. However, if we choose the two $m$-tuples $\left(\delta_{1}, \ldots, \delta_{m}\right)$ and $\left(k_{1}, \ldots, k_{m}\right)$ in Statement 1 in such a way that $m$ and $\left(m n^{*}+k_{1}+\cdots+\right.$ $\left.k_{m}+\delta_{1}+\cdots+\delta_{m}\right) / 2$ are coprime numbers, then it can be easily shown that the corresponding two $m \pi$-periodic solutions are actually subharmonics of order $m$, since their minimal period is exactly $m \pi$. Moreover, Statement 2 implies that the Poincaré map of (1.2) is topologically semi-conjugated to a Bernoulli shift on doubly infinite sequences whose entries belong to a suitable finite set of symbols (see [5] for a similar situation).

Finally, the literature about asymptotically linear periodic problems is very wide; we refer the interested reader to [13] and references contained therein.

The structure of the paper is as follows. In Section 2 we state our main results (see Theorems 2.1 and 2.2), we discuss their optimality and we illustrate an application to a particular case (see Corollary 2.3). In Section 3 we state the result about fixed points of planar maps given in [17]; in Sections 4 and 5 we prove some qualitative properties of the solutions to (1.1) in the intervals of positivity and negativity of $q$, respectively. Finally, the proof of the main theorems is the subject of Section 6 . 


\section{Existence and multiplicity results}

In this section we study the existence and multiplicity of periodic solutions for an equation of the form

$$
\ddot{x}+q(t) g(x)=0,
$$

where the $\pi$-periodic function $q \in C(\mathbf{R})$ and the function $g \in C(\mathbf{R})$ are such that the following conditions hold:

(H1) There exists $\omega \in(0, \pi)$ such that

$$
\begin{aligned}
& q(t) \geq 0, \quad q \not \equiv 0, \quad \forall t \in[0, \omega], \\
& q(t) \leq 0, \quad q \not \equiv 0, \quad \forall t \in[\omega, \pi] .
\end{aligned}
$$

(H2) There exist $m^{+}>0$ and $[a, b] \subset(0, \omega)$ such that

$$
q(t) \geq m^{+}, \quad \forall t \in[a, b] .
$$

and there exist $m^{-}>0$ and $[c, d] \subset(\omega, \pi)$ such that

$$
q(t) \leq-m^{-}, \quad \forall t \in[c, d] .
$$

(H3) The function $g$ is locally Lipschitz and satisfies the sign condition

$$
g(x) x>0, \quad \forall x \in \mathbf{R} \backslash\{0\} .
$$

In what follows, we will make use of the notation

$$
q^{+}=\max \{q(t): t \in[0, \omega]\}
$$

moreover, we define

$$
\hat{g}(\xi, \eta)=\min \{|g(x)|: \xi \leq|x| \leq \eta\}
$$

and

$$
F(\xi, \eta)=\sqrt{2} \sqrt{\frac{\eta-\xi}{\hat{g}(\xi, \eta)}},
$$

for every $(\xi, \eta) \in \mathbf{R}^{2}$ with $0<\xi<\eta$.

With this position, we are able to prove the following results:

Theorem 2.1. Assume that conditions (H1), (H2) and (H3) hold; moreover, suppose that there exist $g_{0}>0, g_{\infty}>0$ and $0<X_{0}<X_{\infty}$ such that

$$
\frac{g(x)}{x} \leq g_{0}, \quad \forall x \in \mathbf{R} \backslash\{0\},|x| \leq X_{0},
$$

and

$$
\frac{g(x)}{x} \geq g_{\infty}, \quad \forall x \in \mathbf{R},|x| \geq X_{\infty}
$$


Let us assume that there exists $M>0$ such that

$$
\left|\frac{g(x)}{x}\right| \leq M, \quad \forall x \in \mathbf{R} \backslash\{0\},
$$

and let us consider $H>0$ such that

$$
x g(x) \geq g_{\infty} x^{2}-H, \quad \forall x \in \mathbf{R} .
$$

Moreover, suppose that there exist $N$ successive natural numbers $n_{1}, \ldots, n_{N} \in \mathbf{N}$ such that

$$
\frac{\omega \sqrt{q^{+} g_{0}}}{\pi} \leq n_{1}<\ldots<n_{N}<\frac{(b-a) \sqrt{m^{+} g_{\infty}}}{\pi}-1
$$

and let us also fix $\epsilon>0$ such that

$$
\frac{\omega \sqrt{q^{+} g_{0}}}{\pi} \leq n_{1}<\ldots<n_{N} \leq \frac{(b-a)(1-\epsilon) \sqrt{m^{+} g_{\infty}}}{\pi}-1 .
$$

Finally, let us assume that

$$
\sqrt{m^{-}}(d-c)>F\left(X_{0} e^{-L \omega}, \sqrt{\frac{H m^{+}}{\epsilon}} \max \left(1,1 / \sqrt{m^{+} g_{\infty}}\right) e^{2 L \omega}\right)
$$

and

$$
\sqrt{m^{-}}(d-c)>F\left(X_{0} e^{-2 L \omega}, \sqrt{\frac{H m^{+}}{\epsilon}} \max \left(1,1 / \sqrt{m^{+} g_{\infty}}\right) e^{L \omega}\right),
$$

where the function $F$ is defined in (2.7) and $L=\max \left(1, q^{+} M\right)$.

Then, the following results hold true:

1. For every $m \in \mathbf{N}$, for every $\delta=\left\{\delta_{1}, \ldots, \delta_{m}\right\} \in\{0,1\}^{m}$ and for every $k=$ $\left\{k_{1}, \ldots, k_{m}\right\} \in\left\{n_{1}, \ldots, n_{N}\right\}^{m}$, with $\delta_{1}+\ldots+\delta_{m}+k_{1}+\ldots+k_{m}$ even, there exist two $m \pi$-periodic solutions $u_{k, \delta}$ and $v_{k, \delta}$ of (2.1) such that

(i) $v_{k, \delta}(0)<0<u_{k, \delta}(0)$ and $\dot{v}_{k, \delta}(0)<0<\dot{u}_{k, \delta}(0)$.

(ii) The solutions $u_{k, \delta}$ and $v_{k, \delta}$ have exactly $k_{j}$ zeros in $((j-1) \pi, \omega+(j-1) \pi)$, $\delta_{j}$ zeros in $(\omega+(j-1) \pi, j \pi)$ and their derivatives have exactly $1-\delta_{j}$ changes of sign in $(\omega+(j-1) \pi, j \pi)$, for every $j=1, \ldots, m$.

2. For every sequence $\delta=\left(\ldots, \delta_{-1}, \delta_{0}, \delta_{1}, \ldots\right)$, with $\delta_{j} \in\{0,1\}$, for every $j \in \mathbf{Z}$, and for every sequence $k=\left(\ldots, k_{-1}, k_{0}, k_{1}, \ldots\right)$, with $k_{j} \in\left\{n_{1}, \ldots, n_{N}\right\}$, for every $j \in \mathbf{Z}$, there exist two bounded solutions $u_{k, \delta}$ and $v_{k, \delta}$ of (2.1) such that

(i) $v_{k, \delta}(0)<0<u_{k, \delta}(0)$ and $\dot{v}_{k, \delta}(0)<0<\dot{u}_{k, \delta}(0)$.

(ii) The solutions $u_{k, \delta}$ and $v_{k, \delta}$ have exactly $k_{j}$ zeros in $((j-1) \pi, \omega+(j-1) \pi)$, $\delta_{j}$ zeros in $(\omega+(j-1) \pi, j \pi)$ and their derivatives have exactly $1-\delta_{j}$ changes of sign in $(\omega+(j-1) \pi, j \pi)$, for every $j \in \mathbf{Z}$. 
Theorem 2.2. Assume that conditions (H1), (H2) and (H3) hold; moreover, suppose that there exist $g_{0}>0, g_{\infty}>0$ and $0<X_{0}<X_{\infty}$ such that

$$
\frac{g(x)}{x} \geq g_{0}, \quad \forall x \in \mathbf{R} \backslash\{0\},|x| \leq X_{0},
$$

and

$$
\frac{g(x)}{x} \leq g_{\infty}, \quad \forall x \in \mathbf{R},|x| \geq X_{\infty} .
$$

Let us assume that there exists $M>0$ such that

$$
\left|\frac{g(x)}{x}\right| \leq M, \quad \forall x \in \mathbf{R} \backslash\{0\},
$$

and let us consider $H>0$ such that

$$
x g(x) \leq g_{\infty} x^{2}+H, \quad \forall x \in \mathbf{R} .
$$

Moreover, suppose that there exist $N$ successive natural numbers $n_{1}, \ldots, n_{N} \in \mathbf{N}$ such that

$$
\frac{\omega \sqrt{q^{+} g_{\infty}}}{\pi}<n_{1}<\ldots<n_{N} \leq \frac{(b-a) \sqrt{m^{+} g_{0}}}{\pi}-1
$$

and let us also fix $\epsilon>0$ such that

$$
\frac{\omega(1+\epsilon) \sqrt{q^{+} g_{\infty}}}{\pi} \leq n_{1}<\ldots<n_{N} \leq \frac{(b-a) \sqrt{m^{+} g_{0}}}{\pi}-1 .
$$

Finally, let us assume that

$$
\sqrt{m^{-}}(d-c)>F\left(X_{0} e^{-L \omega}, \sqrt{\frac{H q^{+}}{\epsilon}} \max \left(1,1 / \sqrt{q^{+} g_{\infty}}\right) e^{2 L \omega}\right)
$$

and

$$
\sqrt{m^{-}}(d-c)>F\left(X_{0} e^{-2 L \omega}, \sqrt{\frac{H q^{+}}{\epsilon}} \max \left(1,1 / \sqrt{q^{+} g_{\infty}}\right) e^{L \omega}\right),
$$

where the function $F$ is defined in (2.7) and $L=\max \left(1, q^{+} M\right)$.

Then, the following results hold true:

1. For every $m \in \mathbf{N}$, for every $\delta=\left\{\delta_{1}, \ldots, \delta_{m}\right\} \in\{0,1\}^{m}$ and for every $k=$ $\left\{k_{1}, \ldots, k_{m}\right\} \in\left\{n_{1}, \ldots, n_{N}\right\}^{m}$, with $\delta_{1}+\ldots+\delta_{m}+k_{1}+\ldots+k_{m}$ even, there exist two $m \pi$-periodic solutions $u_{k, \delta}$ and $v_{k, \delta}$ of (2.1) such that

(i) $v_{k, \delta}(0)<0<u_{k, \delta}(0)$ and $\dot{v}_{k, \delta}(0)<0<\dot{u}_{k, \delta}(0)$.

(ii) The solutions $u_{k, \delta}$ and $v_{k, \delta}$ have exactly $k_{j}$ zeros in $((j-1) \pi, \omega+(j-1) \pi)$, $\delta_{j}$ zeros in $(\omega+(j-1) \pi, j \pi)$ and their derivatives have exactly $1-\delta_{j}$ changes of sign in $(\omega+(j-1) \pi, j \pi)$, for every $j=1, \ldots, m$. 
2. For every sequence $\delta=\left(\ldots, \delta_{-1}, \delta_{0}, \delta_{1}, \ldots\right)$, with $\delta_{j} \in\{0,1\}$, for every $j \in \mathbf{Z}$, and for every sequence $k=\left(\ldots, k_{-1}, k_{0}, k_{1}, \ldots\right)$, with $k_{j} \in\left\{n_{1}, \ldots, n_{N}\right\}$, for every $j \in \mathbf{Z}$, there exist two solutions bounded $u_{k, \delta}$ and $v_{k, \delta}$ of (2.1) such that

(i) $v_{k, \delta}(0)<0<u_{k, \delta}(0)$ and $\dot{v}_{k, \delta}(0)<0<\dot{u}_{k, \delta}(0)$.

(ii) The solutions $u_{k, \delta}$ and $v_{k, \delta}$ have exactly $k_{j}$ zeros in $((j-1) \pi, \omega+(j-1) \pi)$, $\delta_{j}$ zeros in $(\omega+(j-1) \pi, j \pi)$ and their derivatives have exactly $1-\delta_{j}$ changes of sign in $(\omega+(j-1) \pi, j \pi)$, for every $j \in \mathbf{Z}$.

We remark that Theorem 2.1 and Theorem 2.2 still hold when $q$ has a finite number of jump discontinuities. In particular they remain valid if $q$ jumps where it changes sign.

In order to explain our results, let us consider the following example: we deal with the equation

$$
\ddot{x}+\left((\sin (2 t))^{+}-\mu(\sin (2 t))^{-}\right)(x+\lambda \arctan x)=0,
$$

where $\lambda$ and $\mu$ are two positive parameters. We have the following multiplicity result:

Corollary 2.3. It is possible to find $n^{*} \in \mathbf{N}$ and for every $N \in \mathbf{N}$ there exists $\lambda_{N}>1$ such that for every $\lambda \geq \lambda_{N}$ there exists $\mu_{\lambda}>0$ such that for every $\mu \geq \mu_{\lambda}$ we have the following:

1. For all $m \in \mathbf{N}$, for all $\delta=\left(\delta_{1}, \ldots, \delta_{m}\right) \in\{0,1\}^{m}$ and for all $\left(k_{1}, \ldots, k_{m}\right) \in$ $\{1, \ldots, N\}$ such that $m n^{*}+k_{1}+\cdots+k_{m}+\delta_{1}+\cdots+\delta_{m}$ is even, there are two $m \pi$-periodic solutions of equation (2.24) which have exactly $n^{*}+k_{j}$ zeros in $((j-1) \pi,(j-1) \pi+\pi / 2)$ and $\delta_{j}$ zeros in $((j-1) \pi+\pi / 2, j \pi)$, for every $j=1, \ldots, m$;

2. For any pair of sequences $\delta=\left(\ldots, \delta_{-1}, \delta_{0}, \delta_{1}, \ldots\right) \in\{0,1\}^{\mathbf{Z}}$ and $k=$ $\left(\ldots, k_{-1}, k_{0}, k_{1}, \ldots\right) \in\{1, \ldots, N\}^{\mathbf{Z}}$ there are two bounded solutions of (2.24) which have exactly $n^{*}+k_{j}$ zeros in $((j-1) \pi,(j-1) \pi+\pi / 2)$ and $\delta_{j}$ zeros in $((j-1) \pi+\pi / 2, j \pi)$, for every $j \in \mathbf{Z}$

( $n^{*}$ is a natural number which does not depend on $N, \lambda$ and $\mu$ ).

Proof. We apply Theorem 2.2. We are in the case when $g(x)=g_{\lambda}(x)=x+$ $\lambda \arctan x$, for every $x \in \mathbf{R}$ and $q(t)=(\sin (2 t))^{+}-\mu(\sin (2 t))^{-}$, for every $t \in[0, \pi]$, with $\omega=\pi / 2$ and $q^{+}=1$.

Let us fix $\tau \in(0,1 / 2)$; then, setting $g_{0}=1+\lambda-\tau$ and $g_{\infty}=1+\tau$, it is easy to see that there exist $X_{0}=X_{0}(\tau)<X_{\infty}(\tau)=X_{\infty}$ such that (2.16) and (2.17) hold true.

We now fix $\alpha \in(0, \pi / 4)$ and take $[a, b],[c, d], m^{+}$and $m^{-}$as in (2.2) and (2.3), with $[a, b]=[\alpha, \pi / 2-\alpha],[c, d]=[\pi / 2+\alpha, \pi-\alpha]$ and $m^{+}=\sin (2 \alpha)$, $m^{-}=\mu \sin (2 \alpha)$.

Now, let $n^{*}$ be the greatest integer such that $n^{*} \leq \frac{1}{2} \sqrt{1+\tau}$ and, for every $N \in \mathbf{N}$, let us consider $\lambda_{N}>1$ such that

$$
\left(\frac{1}{2}-\frac{2 \alpha}{\pi}\right) \sqrt{\sin (2 \alpha)} \sqrt{1+\lambda_{N}-\tau}-1-\frac{1}{2} \sqrt{1+\tau}>N .
$$


As a consequence we have that the numbers $n^{*}+1, \ldots, n^{*}+N$ satisfy (2.20) and (2.21), for a sufficiently small $\epsilon>0$. For every $\lambda \geq \lambda_{N}$ we consider the constants $M=M(\lambda)$ and $H=H(\lambda)$ given in (2.18) and (2.19), respectively. Finally, we denote by $F_{\lambda}$ the maximum between the right members of (2.22) and (2.23).

Let us now take $\mu=\mu_{\lambda}$ such that

$$
\sqrt{\mu}\left(\frac{\pi}{2}-2 \alpha\right) \sqrt{\sin (2 \alpha)}>F_{\lambda}
$$

For every $\lambda \geq \lambda_{N}$ and for every $\mu \geq \mu_{\lambda}$ all the assumptions of Theorem 2.2 are fulfilled. An application of the first statement of Theorem 2.2 proves the result.

Let us consider now the following example in order to discuss the sharpness of Theorem 2.1 and Theorem 2.2: let us study the equation

$$
\ddot{x}+\bar{q}(t) \bar{g}(x)=0
$$

where $\bar{q}: \mathbf{R} \longrightarrow \mathbf{R}$ is a $\pi$-periodic function such that

$$
\bar{q}(t)= \begin{cases}1 & \text { if } 0 \leq t<\frac{\pi}{2} \\ -\mu & \text { if } \frac{\pi}{2} \leq t<\pi\end{cases}
$$

and $\bar{g}: \mathbf{R} \longrightarrow \mathbf{R}$ is defined by

$$
\bar{g}(x)= \begin{cases}\alpha^{2} x & \text { if }|x| \leq 1 \\ \beta^{2} x-\beta^{2}+\alpha^{2} & \text { if } x>1 \\ \beta^{2} x+\beta^{2}-\alpha^{2} & \text { if } x<-1\end{cases}
$$

with $\mu>0$ and $\beta>\alpha>0$. Let us see which conditions we have to impose on $\alpha$, $\beta$ and $\mu$ if we wish to find $\pi$-periodic solutions of (2.26) with exactly one zero in $(0, \pi / 2)$ and another one in $(\pi / 2, \pi)$ by applying Statement 1 of Theorem 2.1 with $m=n_{1}=\delta_{1}=1$. Clearly we have $\bar{g}(x) / x \leq M=\beta^{2}$ for all $x$ and:

$$
\frac{\bar{g}(x)}{x}=g_{0}=\alpha^{2} \forall|x| \leq X_{0}=1 \quad \text { and } \quad \frac{\bar{g}(x)}{x} \geq g_{\infty}=\beta^{2}-\frac{\beta^{2}-\alpha^{2}}{X_{\infty}} \forall|x| \geq X_{\infty},
$$

with $X_{\infty}>1$ to be suitably chosen. Moreover we can set:

$$
q^{+}=m^{+}=1, \quad m^{-}=\mu, \quad a=0, \quad b=c=\omega=\frac{\pi}{2}, \quad d=\pi .
$$

Therefore condition (2.12) with $N=1$ and $n_{1}=1$ implies:

$$
\alpha \leq 2 \quad \text { and } \quad \beta^{2}-\frac{\beta^{2}-\alpha^{2}}{X_{\infty}}>16
$$


in particular, $\beta$ must be strictly greater than 4 and for every such a $\beta$ it is always possible to find $X_{\infty}>1$ such that the second inequality is satisfied. Hence let us choose $\alpha=2$ and $\beta=5$ in such a way that we obtain for $X_{\infty}$ the constraint:

$$
X_{\infty}>\frac{\beta^{2}-\alpha^{2}}{\beta^{2}-16}=\frac{21}{9} .
$$

From this it can be shown that the best choices for $H$ in (2.11) and $\epsilon$ in (2.13) are

$$
H=\frac{21}{4} X_{\infty} \quad \text { and } \quad \epsilon=1-\sqrt{\frac{16 X_{\infty}}{25 X_{\infty}-21}},
$$

so that conditions (2.14) and (2.15) imply that $\mu>f\left(X_{\infty}\right)$, where $f:(21 / 9,+\infty)$ $\rightarrow \mathbf{R}$ has a positive global minimum in $X_{\infty}^{\text {best }}$. Therefore Theorem 2.1 provides the desired pair of solutions only if $\mu$ is larger than $f\left(X_{\infty}^{\text {best }}\right)$.

However, straightforward but cumbersome calculations can be performed by solving explicitly equation (2.26) and it can be shown that if $\alpha \leq 2$ and $\beta>4$ there are exactly two $\pi$-periodic solution with one zero in $(0, \pi / 2)$ and another one in $(\pi / 2, \pi)$ and that this happens for every $\mu>0$. Hence the number of solutions with prescribed nodal behavior, which we find via Theorem 2.1 and Theorem 2.2, is optimal with respect to our set of assumptions. On the other hand, the generality of the functions $q$ and $g$ that we allow forces us to adopt estimates, such as those of Lemma 4.1 and Lemma 5.1, which imply conditions such as (2.14)-(2.15) and (2.22)-(2.23) that look rough when applied to simple cases as (2.26).

The proof of Theorem 2.1 and Theorem 2.2 will follow from the application of the abstract result (that is due to F. Zanolin and the second author [17]) which is illustrated in the next section. We just stress the fact that Statement 2 in Theorem 2.1, Theorem 2.2, and Theorem 3.1 can be interpreted as a result about the presence of chaos in the sense of coin tossing [10]. See [17] for more details.

In the rest of the paper, we will consider the planar system

$$
\left\{\begin{array}{l}
\dot{x}=y \\
\dot{y}=-q(t) g(x)
\end{array}\right.
$$

equivalent to (2.1). We will denote by $z=(x, y)$ any solution to (2.27) and, for every $p \in \mathbf{R}^{2}$ and for every $t_{0} \in \mathbf{R}$, by $z\left(\cdot ; t_{0}, p\right)$ the unique solution to the Cauchy problem

$$
\left\{\begin{array}{l}
\dot{x}=y \\
\dot{y}=-q(t) g(x) \\
\left(x\left(t_{0}\right), y\left(t_{0}\right)\right)=p .
\end{array}\right.
$$

We observe that the assumptions on $g$ guarantee that $z$ is globally defined. Moreover, we shall use polar coordinates $(\theta(t), \rho(t))$ to represent $z\left(t ; t_{0}, p\right)$ for every $p \neq 0$. Indeed, for every $p \in \mathbf{R}^{2} \backslash\{0\}$, we can associate to $z$ polar coordinates such that $z\left(t ; t_{0}, p\right)=(\rho(t) \cos \theta(t), \rho(t) \sin \theta(t))$ and define the normalized angular displacement (rotation number)

$$
\operatorname{rot}_{\left[s_{1}, s_{2}\right]}(p):=\frac{\theta\left(s_{1}\right)-\theta\left(s_{2}\right)}{\pi}
$$


for every interval $\left[s_{1}, s_{2}\right] \subset \mathbf{R}$. We also recall (see e.g. [7]) that the rotation number can be computed by means of the integral formula

$$
\operatorname{rot}_{\left[s_{1}, s_{2}\right]}(p)=\frac{\mu}{\pi} \int_{s_{1}}^{s_{2}} \frac{q(t) x(t) g(x(t))+x^{\prime}(t)^{2}}{\mu^{2} x(t)^{2}+x^{\prime}(t)^{2}} d t,
$$

for every $\mu>0$.

\section{Fixed points of planar maps}

In order to present the abstract result on the existence of fixed points of planar maps, we need some preliminary definitions.

For $0<r_{1}<r_{2}$, let us consider the closed annulus

$$
\mathcal{A}\left[r_{1}, r_{2}\right]:=\left\{z \in \mathbf{R}^{2}: r_{1} \leq|z| \leq r_{2}\right\}
$$

and, for $r>0$, the circumference $C_{r}:=\left\{z \in \mathbf{R}^{2}:|z|=r\right\}$.

Moreover, for any unit vectors $v=\left(v_{1}, v_{2}\right)$ and $w=\left(w_{1}, w_{2}\right)$ of the plane with $v \neq w$, we denote by $\ell_{v}=\{t v: t>0\}$ and $\ell_{w}=\{t w: t>0\}$ the (open) half-lines from the origin through $v$ and $w$. The 0-pointed angle $\widehat{v 0 w}$ is the union of $\ell_{v}, \ell_{w}$ with all the half-lines $\ell_{u}$ such that the triple $(v, u, w)$ is positively oriented.

For $r_{1}$ and $r_{2}$ with $0<r_{1}<r_{2}$, we define the (closed) set

$$
[\widehat{v 0 w}]_{r_{1}}^{r_{2}}:=\left\{z \in \widehat{v 0 w}: r_{1} \leq|z| \leq r_{2}\right\}=\widehat{v 0 w} \cap \mathcal{A}\left[r_{1}, r_{2}\right] \text {. }
$$

We say that $[\widehat{v 0 w}]_{r_{1}}^{r_{2}}$ is a conical shell if it is determined by a pointed angle $\widehat{v 0 w}$ at the origin such that $\overline{v 0 w}$ is convex and also $w \neq-v$.

Now, let us consider a continuous map $\psi: \mathbf{R}^{2} \supset \operatorname{dom}(\psi):=D_{\psi} \longrightarrow \mathbf{R}^{2}$ and a set $\mathscr{D} \subset D_{\psi}$. We say that the (continuous) map is proper on $\mathscr{D}$ if $\psi^{-1}(\mathcal{K}) \cap \mathscr{D}$ is compact for each compact set $\mathcal{K} \subset \mathbf{R}^{2}$. In this situation, we will also say that the pair $(\mathscr{D}, \psi)$ is proper. Given a map $\psi$ and a set $\mathscr{D} \subset D_{\psi}$, we also define $(\mathscr{D}, \psi)$ proper on compact sets if, for each $K$ compact, $\psi$ is proper on $\mathscr{D} \cap K$.

We consider two conical shells

$$
\boldsymbol{W}_{0}:=\left[\widehat{v_{0} 0 w_{0}}\right]_{r_{1}^{0}}^{r_{2}^{0}}, \quad \mathcal{W}_{1}:=\left[\widehat{v_{1} 0 w_{1}}\right]_{r_{1}^{1}}^{r_{2}^{1}},
$$

which we assume to be disjoint, and we denote by $\psi^{j}$ the $j$ th iterate of $\psi$, with the convention that $\psi^{1}=\psi$.

Finally, if $\psi: \mathbf{R}^{2} \supset \operatorname{dom}(\psi):=D_{\psi} \longrightarrow \mathbf{R}^{2}$ is a continuous function, we define any continuous map $\eta:\left(\mathcal{W}_{0} \cup \mathcal{W}_{1}\right) \cap D_{\psi} \rightarrow \mathbf{R}$, as a tag associated to $\psi$. The pair $(\psi, \eta)$ will be called a tagged map.

We are now in position to state the following abstract result (see [17]): 
Theorem 3.1 ([17, Theorem 4]). Let $\mathscr{D} \subset D_{\psi}$ and let $(\psi, \eta)$ be a tagged map with $(\mathscr{D}, \psi)$ proper on the compact sets. For $\mathcal{W}_{0}$ and $\mathcal{W}_{1}$ as above, let us suppose that for every $i, j \in\{0,1\}$ there is a (non-empty) set of indices $\mathcal{N}_{(i, j)} \subset \mathbf{N}$ such that, for each $k \in \mathcal{N}_{(i, j)}$ there is a compact interval $\Lambda^{k}=\Lambda_{(i, j)}^{k} \subset \mathbf{R}$, with $\Lambda^{n} \cap \Lambda^{m}=\emptyset$ for $n \neq m$, and such that any path $\sigma$ contained in $\mathcal{W}_{i}$ and intersecting $C_{r_{1}^{i}}$ and $C_{r_{2}^{i}}$, contains a sub-path $\sigma_{(i, j)}^{k} \subset \mathcal{D}$ such that

$$
\psi\left(\sigma_{(i, j)}^{k}\right) \subset \widehat{v_{j} 0 w_{j}}, \quad \eta\left(\sigma_{(i, j)}^{k}\right) \subset \Lambda^{k}
$$

and

$$
\psi\left(\sigma_{(i, j)}^{k}\right) \cap C_{r_{1}^{j}} \neq \emptyset, \quad \psi\left(\sigma_{(i, j)}^{k}\right) \cap C_{r_{2}^{j}} \neq \emptyset .
$$

Then the following conclusions hold:

1. For every $m \geq 1$, for every sequence $\delta^{\prime}=\left(\delta_{1}^{\prime}, \ldots, \delta_{m}^{\prime}\right)$, with $\delta_{j}^{\prime} \in\{0,1\}$ (for each $j=1, \ldots, m)$ and for every sequence $\kappa=\left(k_{1}, \ldots, k_{m}\right)$, with

$$
k_{1} \in \mathcal{N}_{\left(\delta_{m}^{\prime}, \delta_{1}^{\prime}\right)}, k_{2} \in \mathcal{N}_{\left(\delta_{1}^{\prime}, \delta_{2}^{\prime}\right)}, \ldots, k_{m} \in \mathcal{N}_{\left(\delta_{m-1}^{\prime}, \delta_{m}^{\prime}\right)},
$$

there exists a fixed point $\tilde{p}_{\left(\kappa, \delta^{\prime}\right)} \in \mathscr{D} \cap \mathcal{W}_{\delta_{m}^{\prime}}$ of $\psi^{m}$ which satisfies

$$
\psi^{j}\left(\tilde{p}_{\left(\kappa, \delta^{\prime}\right)}\right) \in \mathcal{W}_{\delta_{j}^{\prime}}, \quad \eta\left(\psi^{j}\left(\tilde{p}_{\left(\kappa, \delta^{\prime}\right)}\right)\right) \in \Lambda_{\left(\delta_{j-1}^{\prime}, \delta_{j}^{\prime}\right)}^{k_{j}}, \quad \forall j=1, \ldots, m
$$

(we use here the convention that $\delta_{0}^{\prime}=\delta_{m}^{\prime}$ ).

2. For every doubly-infinite sequence $\delta^{\prime}=\left(\ldots, \delta_{-2}^{\prime}, \delta_{-1}^{\prime}, \delta_{0}^{\prime}, \delta_{1}^{\prime}, \delta_{2}^{\prime}, \ldots\right)$, with $\delta_{j}^{\prime} \in\{0,1\}(\forall j \in \mathbf{Z})$ and for every $\kappa=\left(\ldots, k_{-2}, k_{-1}, k_{0}, k_{1}, k_{2}, \ldots\right)$, with

$$
k_{m} \in \mathcal{N}_{\left(\delta_{m-1}^{\prime}, \delta_{m}^{\prime}\right)}, \quad(\forall m \in \mathbf{Z}),
$$

there exists a double-sided sequence of points

$$
\tilde{p}_{\left(\kappa, \delta^{\prime}\right)}=\left(\ldots, \tilde{p}_{-2}, \tilde{p}_{-1}, \tilde{p}_{0}, \tilde{p}_{1}, \tilde{p}_{2}, \ldots\right),
$$

with $\tilde{p}_{j} \in \mathscr{D} \cap \mathcal{W}_{\delta_{j}^{\prime}}$ such that

$$
\psi\left(\tilde{p}_{j}\right)=\tilde{p}_{j+1}, \quad \eta\left(\psi^{j}\left(\tilde{p}_{j}\right)\right) \in \Lambda_{\left(\delta_{j-1}^{\prime}, \delta_{j}^{\prime}\right)}^{k_{j}}, \quad \forall j \in \mathbf{Z} .
$$

\section{Behavior on the intervals of positivity of $q$}

In this section we study the behavior of the solutions to $(2.1)$ in the interval $[0, \omega]$ where $q$ is non-negative. We first give a preliminary lemma on the solutions to the system (2.27) on the interval $[0, \omega]$ :

Lemma 4.1. Under the assumptions of Theorem 2.1 or Theorem 2.2, for every solution $z$ of (2.27) we have

$$
|z(t)| \leq e^{L|t-s|}|z(s)|, \quad \forall t, s \in[0, \omega],
$$

where $L=\max \left(1, q^{+} M\right)$. 
Proof. We first observe that it is sufficient to prove (4.1) when $t \geq s$. Indeed, the case $t<s$ can be reduced to the other by means of the change of variable $t \mapsto-t$.

For every $t \geq s$ we have

$$
z(t)=z(s)+\int_{s}^{t}(y(\tau),-q(\tau) g(x(\tau))) d \tau ;
$$

therefore, using (2.10), we obtain

$$
\begin{aligned}
|z(t)| & \leq|z(s)|+\int_{s}^{t} \sqrt{y(\tau)^{2}+\left(q^{+}\right)^{2} M^{2} x(\tau)^{2}} d \tau \\
& \leq|z(s)|+\max \left(1, q^{+} M\right) \int_{s}^{t}|z(\tau)| d \tau .
\end{aligned}
$$

An application of Gronwall's Lemma proves the result.

By means of Lemma 4.1, we easily obtain the following result:

Proposition 4.2. Under the assumptions of Theorem 2.1 or Theorem 2.2, we have:

1. For every $A>0$ there exists $B=A e^{-L \omega}$ such that for every solution $z$ to (2.27) we have

$$
\begin{aligned}
& \exists t_{0} \in[0, \omega]:\left|z\left(t_{0}\right)\right| \leq B \quad \Rightarrow \quad|z(t)| \leq A, \quad \forall t \in[0, \omega] . \\
& \exists t_{0} \in[0, \omega]:\left|z\left(t_{0}\right)\right| \geq A \quad \Rightarrow \quad|z(t)| \geq B, \quad \forall t \in[0, \omega] .
\end{aligned}
$$

2. For every $C>0$ there exists $D=C e^{L \omega}$ such that for every solution $z=(x, y)$ to (2.27) and for every $\mu>0$ we have

$$
\begin{aligned}
& \exists t_{0} \in[0, \omega]:\left|z\left(t_{0}\right)\right| \leq C \Rightarrow \sqrt{\mu^{2} x(t)^{2}+x^{\prime}(t)^{2}} \leq D \max (\mu, 1), \forall t \in[0, \omega] . \\
& \exists t_{0} \in[0, \omega]:\left|z\left(t_{0}\right)\right| \geq D \max (1,1 / \mu) \Rightarrow \sqrt{\mu^{2} x(t)^{2}+x^{\prime}(t)^{2}} \geq C, \forall t \in[0, \omega] .
\end{aligned}
$$

We are able to prove the following results on the asymptotic behavior of the rotation number defined in (2.29):

Proposition 4.3. Suppose that the assumptions of Theorem 2.1 hold true. Then there exists $R_{1}=X_{0} e^{-L \omega}$ such that for every non-trivial solution $z$ to (2.27) we have

$$
|z(0)| \leq R_{1} \Rightarrow \operatorname{rot}_{[0, \omega]}(z(0)) \leq \frac{\omega}{\pi} \sqrt{q^{+} g_{0}} .
$$

Proof. We first observe that, given $X_{0}$ as in assumption (2.8), by the first statement of Proposition 4.2 we can find $R_{1}=X_{0} e^{-L \omega}$ such that

$$
|z(0)| \leq R_{1} \quad \Rightarrow \quad|z(t)| \leq X_{0}, \quad \forall t \in[0, \omega] .
$$

Now, let $z$ be a solution to (2.27) such that $|z(0)| \leq R_{1}$. From (2.8) and (4.4), we infer that

$$
x(t) g(x(t)) \leq g_{0} x(t)^{2}, \quad \forall t \in[0, \omega] .
$$


Using (2.30), with $\mu=\sqrt{q^{+} g_{0}}$, and (4.5) we obtain

$$
\begin{aligned}
\operatorname{rot}_{[0, \omega]}(z(0)) & =\frac{\sqrt{q^{+} g_{0}}}{\pi} \int_{0}^{\omega} \frac{q(t) x(t) g(x(t))+x^{\prime}(t)^{2}}{q^{+} g_{0} x(t)^{2}+x^{\prime}(t)^{2}} d t \leq \\
& \leq \frac{\sqrt{q^{+} g_{0}}}{\pi} \int_{0}^{\omega} \frac{q^{+} g_{0} x(t)^{2}+x^{\prime}(t)^{2}}{q^{+} g_{0} x(t)^{2}+x^{\prime}(t)^{2}} d t \leq \frac{\omega}{\pi} \sqrt{q^{+} g_{0}} .
\end{aligned}
$$

This concludes the proof.

In an analogous way it is possible to prove the validity of the following:

Proposition 4.4. Suppose that the assumptions of Theorem 2.2 hold true. Then there exists $R_{1}=X_{0} e^{-L \omega}$ such that for every non-trivial solution $z$ to (2.27) we have

$$
|z(0)| \leq R_{1} \Rightarrow \operatorname{rot}_{[0, \omega]}(z(0)) \geq \frac{b-a}{\pi} \sqrt{m^{+} g_{0}} .
$$

Proposition 4.5. Suppose that the assumptions of Theorem 2.1 hold true. Then there exists $R_{2}=e^{L \omega} \sqrt{\mathrm{Hm}^{+} / \epsilon} \max \left(1,1 / \sqrt{m^{+} g_{\infty}}\right)$ such that for every non-trivial solution $z$ to (2.27) we have

$$
|z(0)| \geq R_{2} \Rightarrow \operatorname{rot}_{[0, \omega]}(z(0)) \geq \frac{b-a}{\pi}(1-\epsilon) \sqrt{m^{+} g_{\infty}} .
$$

Proof. Since

$$
\operatorname{rot}_{[0, \omega]}(z(0)) \geq \operatorname{rot}_{[a, b]}(z(0)),
$$

it is sufficient to show that

$$
\operatorname{rot}_{[a, b]}(z(0)) \geq \frac{b-a}{\pi}(1-\epsilon) \sqrt{m^{+} g_{\infty}} .
$$

Let us fix $\mu=\sqrt{m^{+} g_{\infty}}$ and $C=\sqrt{H m^{+} / \epsilon}$, where $H$ and $\epsilon$ are given in (2.11) and (2.13), respectively. An application of the second statement of Proposition 4.2 gives the existence of a number $D=C e^{L \omega}$ such that for every solution $z$ to (2.27) we have

$$
\begin{aligned}
|z(0)| \geq D \max \left(1,1 / \sqrt{m^{+} g_{\infty}}\right) \Rightarrow m^{+} g_{\infty} x(t)^{2}+x^{\prime}(t)^{2} & \geq \frac{H m^{+}}{\epsilon}, \\
& \forall t \in[a, b] .
\end{aligned}
$$

Let us set $R_{2}=D \max \left(1,1 / \sqrt{m^{+} g_{\infty}}\right)$ and let $z$ be a solution to (2.27) such that $|z(0)| \geq R_{2}$. Using (2.30) with the previous choice of $\mu$, recalling (2.11) and (4.6), we deduce that 


$$
\begin{aligned}
& \operatorname{rot}_{[a, b]}(z(0))= \\
& \quad=\frac{\sqrt{m^{+} g_{\infty}}}{\pi} \int_{a}^{b} \frac{q(t) x(t) g(x(t))+x^{\prime}(t)^{2}}{m^{+} g_{\infty} x(t)^{2}+x^{\prime}(t)^{2}} d t \geq \\
& \quad \geq \frac{\sqrt{m^{+} g_{\infty}}}{\pi}\left(\int_{a}^{b} \frac{m^{+} g_{\infty} x(t)^{2}+x^{\prime}(t)^{2}}{m^{+} g_{\infty} x(t)^{2}+x^{\prime}(t)^{2}} d t-\int_{a}^{b} \frac{H m^{+}}{m^{+} g_{\infty} x(t)^{2}+x^{\prime}(t)^{2}} d t\right) \geq \\
& \quad \geq \frac{b-a}{\pi}(1-\epsilon) \sqrt{m^{+} g_{\infty}} .
\end{aligned}
$$

The proof is complete.

In a similar way it is possible to prove the following:

Proposition 4.6. Suppose that the assumptions of Theorem 2.2 hold true. Then there exists $R_{2}=e^{L \omega} \sqrt{H q^{+} / \epsilon} \max \left(1,1 / \sqrt{q^{+} g_{\infty}}\right)$ such that for every non-trivial solution $z$ to (2.27) we have

$$
|z(0)| \geq R_{2} \Rightarrow \operatorname{rot}_{[0, \omega]}(z(0)) \leq \frac{\omega}{\pi}(1+\epsilon) \sqrt{q^{+} g_{\infty}} .
$$

Finally, we give the following result, which is a straightforward consequence of Proposition 4.2:

Proposition 4.7. For every $R_{1}<R_{2}$ there exist $R_{1}^{\prime}=R_{1} e^{-L \omega}$ and $R_{2}^{\prime}=R_{2} e^{L \omega}$ such that for every solution $z$ to (2.27) we have

$$
R_{1} \leq|z(0)| \leq R_{2} \quad \Rightarrow \quad R_{1}^{\prime} \leq|z(\omega)| \leq R_{2}^{\prime}
$$

\section{Behavior on the intervals of negativity of $q$}

In this section we study the behavior of the solutions to (2.1) in the interval $[\omega, \pi]$ where $q$ is negative. To this end, we consider four positive numbers $R_{1}, R_{2}, R_{1}^{\prime}$ and $R_{2}^{\prime}$ as in Proposition 4.7. We are able to prove the following result:

Lemma 5.1. Let us consider $m^{-}$and $[c, d]$ as in assumption (2.3) and the function $F$ defined in (2.7). Then the following relations hold true:

$$
\begin{aligned}
& \sqrt{m^{-}}(d-c)>F\left(R_{1}, R_{2}^{\prime}\right) \Rightarrow\left\{\begin{array}{l}
\left|z\left(\omega ; \pi,\left( \pm R_{1}, 0\right)\right)\right|>R_{2}^{\prime} ; \\
\left|z\left(\omega ; \pi,\left(0, \pm R_{1}\right)\right)\right|>R_{2}^{\prime} ;
\end{array}\right. \\
& \sqrt{m^{-}}(d-c)>F\left(R_{1}^{\prime}, R_{2}\right) \Rightarrow \begin{cases}\left|z\left(\pi ; \omega,\left(p_{1}, 0\right)\right)\right|>R_{2}, & \forall\left|p_{1}\right|>R_{1}^{\prime} ; \\
\left|z\left(\pi ; \omega,\left(0, p_{1}\right)\right)\right|>R_{2}, & \forall\left|p_{1}\right|>R_{1}^{\prime} .\end{cases}
\end{aligned}
$$

Proof. Let us start with the first inequality in (5.1) and suppose that $\left|z\left(\omega ; \pi,\left(R_{1}, 0\right)\right)\right| \leq R_{2}^{\prime}$. For simplicity, let $x(t)=x\left(t ; \pi,\left(R_{1}, 0\right)\right)$. By the sign condition on $q$ and $g$ and the fact that $x(\pi)=R_{1}>0$ and $\dot{x}(\pi)=0$, it is easy to see that $x(t) \geq R_{1}$ and $\dot{x}(t) \leq 0$ for every $t \in[\omega, \pi]$. 
Now, from assumption (2.3) and the previous discussion, we obtain

$$
\dot{x}(t) \ddot{x}(t)=-q(t) g(x(t)) \dot{x}(t) \leq m^{-} g(x(t)) \dot{x}(t), \quad \forall t \in[c, d] .
$$

By integrating (5.3) between $t$ and $d$, we get

$$
\frac{1}{2} \dot{x}(d)^{2}-\frac{1}{2} \dot{x}(t)^{2} \leq m^{-}(G(x(d))-G(x(t))), \quad \forall t \in[c, d],
$$

where $G(x)=\int_{0}^{x} g(s) d s$; therefore we have

$$
-\frac{\dot{x}(t)}{\sqrt{(G(x(d))-G(x(t)))}} \geq \sqrt{2 m^{-}}, \quad \forall t \in[c, d] .
$$

We now integrate (5.5) from $c$ to $d$ to deduce that

$$
\sqrt{2 m^{-}}(d-c) \leq \int_{x(d)}^{x(c)} \frac{d \xi}{\sqrt{(G(\xi)-G(x(d)))}} .
$$

We then observe that, by the intermediate value theorem, there exists $x_{\xi} \in$ $(x(d), x(c))$ such that

$$
(G(\xi)-G(x(d)))=g\left(x_{\xi}\right)(\xi-x(d)) .
$$

Hence, from (5.6) and (5.7), recalling also the definition of $\hat{g}$ given in (2.6), we infer that

$$
\sqrt{2 m^{-}}(d-c) \leq \frac{1}{\sqrt{\hat{g}\left(R_{1}, R_{2}^{\prime}\right)}} \int_{x(d)}^{x(c)} \frac{d \xi}{\sqrt{\xi-x(d)}} \leq \frac{2}{\sqrt{\hat{g}\left(R_{1}, R_{2}^{\prime}\right)}} \sqrt{R_{2}^{\prime}-R_{1}} .
$$

This concludes the proof of the first part of (5.1).

By means of similar computations it can be shown that also the other inequalities in (5.1) and (5.2) hold true.

\section{Proof of the main results}

In this section we give the details of the proof of Theorem 2.1; the proof of Theorem 2.2 can be obtained in the same way using Proposition 4.4 and Proposition 4.6 instead of Proposition 4.3 and Proposition 4.5, respectively.

In order to prove the result we apply Theorem 3.1 as already observed. To this end, we need to define the maps $\psi$ and $\eta$ and the sets $\mathcal{W}_{0}$ and $\mathcal{W}_{1}$. Indeed, $\psi$ is the Poincaré map associated to $(2.27)$ in $[0, \pi]$, i.e.

$$
\psi(p)=z(\pi ; 0, p), \quad \forall p \in \mathbf{R}^{2}=\mathscr{D}_{\psi} .
$$

We observe (see also [17, Example 3]) that the global continuability of solutions to (2.27) ensures that $\left(\mathbf{R}^{2}, \psi\right)$ is proper on compact sets. Moreover, let us consider the numbers $R_{1}$ and $R_{2}$ given in Proposition 4.3 and Proposition 4.5, respectively, and the corresponding numbers $R_{1}^{\prime}$ and $R_{2}^{\prime}$ as in Proposition 4.7. We then take 
the conical shells $\mathcal{W}_{0}$ and $\mathcal{W}_{1}$ as in (3.1) with $r_{1}^{0}=r_{1}^{1}=R_{1}, r_{2}^{0}=r_{2}^{1}=R_{2}$, $v_{0}=(1,0)=-v_{1}$ and $w_{0}=(0,1)=-w_{1}$.

For every $p \in \mathcal{W}_{0} \cup \mathcal{W}_{1}$ we finally define

$$
\eta(p)=\vartheta(p)-\pi \operatorname{rot}_{[0, \omega]}(p),
$$

where $\vartheta(p) \in[-\pi, \pi / 2]$ is the continuous angular coordinate of $p$.

Let us take

$$
\mathcal{N}_{(i, j)}=\mathcal{N}=\left\{n_{1}, \ldots, n_{N}\right\}
$$

and, moreover,

$$
\Lambda_{(i, j)}^{k}=\left[-(k+i) \pi-\frac{\pi}{2},-(k+i) \pi\right] \quad \forall k \in \mathcal{N} .
$$

We observe that if there exist $p \in \mathcal{W}_{i}$ and $k \in \mathcal{N}$ such that $\eta(p)$ belongs to $\Lambda_{(i, j)}^{k}$ then $x(\cdot ; 0, p)$ has exactly $k$ zeros in $(0, \omega)$.

Let us fix a path $\sigma:[0,1] \longrightarrow \mathcal{W}_{i}$ such that $|\sigma(0)|=R_{1}$ and $|\sigma(1)|=R_{2}$. Using Proposition 4.3 we deduce that

$$
\eta(\sigma(0))=\vartheta(\sigma(0))-\pi \operatorname{rot}_{[0, \omega]}(\sigma(0)) \geq-i \pi-\omega \sqrt{q^{+} g_{0}} .
$$

On the other hand, from Proposition 4.5 we infer that

$$
\eta(\sigma(1))=\vartheta(\sigma(1))-\pi \operatorname{rot}_{[0, \omega]}(\sigma(1)) \leq-i \pi+\frac{\pi}{2}-(b-a)(1-\epsilon) \sqrt{m^{+} g_{\infty}} .
$$

Hence, recalling (2.13), we obtain that

$$
\left[-\left(n_{N}+i\right) \pi-\frac{\pi}{2},-\left(n_{1}+i\right) \pi\right] \subset \eta(\sigma([0,1])) .
$$

Therefore, for every $k \in \mathcal{N}$ we find $\left[s_{k}, s_{k}^{\prime}\right] \subset[0,1]$ such that

$$
\eta\left(\sigma\left(\left[s_{k}, s_{k}^{\prime}\right]\right)\right)=\Lambda_{(i, j)}^{k}
$$

and

$$
\eta\left(\sigma\left(s_{k}\right)\right)=-\left(n_{k}+i\right) \pi, \quad \eta\left(\sigma\left(s_{k}^{\prime}\right)\right)=-\left(n_{k}+i\right) \pi-\frac{\pi}{2} .
$$

We now define $\gamma(s)=z(\omega ; 0, \sigma(s))$, for every $s \in[0,1]$. We observe that, from Proposition 4.7, we also have

$$
R_{1}^{\prime} \leq|\gamma(s)| \leq R_{2}^{\prime}, \quad \forall s \in[0,1] .
$$

Moreover, $\gamma(s)$ belongs to the fourth (second) quadrant if $k$ is even (odd, respectively). For simplicity, assume that $k$ is even.

We first consider the point $F_{1}=\gamma\left(s_{k}\right)$; by the sign conditions on $q$ in $[\omega, \pi]$ and $g$, it is easy to see that $z\left(\pi ; \omega, \gamma\left(s_{k}\right)\right)=z\left(\pi ; 0, \sigma\left(s_{k}\right)\right)$ belongs to the first quadrant. Moreover, from (5.2) we deduce that $\left|z\left(\pi ; \omega, \gamma\left(s_{k}\right)\right)\right| \geq R_{2}$. 
Let us now define the curve $\gamma^{\prime}(s)=z(\omega ; \pi,(s, 0))$ for $s \in\left[0, R_{1}\right]$. Again, by the sign conditions, we infer that $\gamma^{\prime}(s)$ belongs to the fourth quadrant, for every $s \in\left[0, R_{1}\right]$. Moreover, we have that $\gamma^{\prime}(0)=(0,0)$ and, by $(5.1),\left|\gamma^{\prime}\left(R_{1}\right)\right|>R_{2}^{\prime}$. Therefore, the images of $\left.\gamma\right|_{\left[s_{k}, s_{k}^{\prime}\right]}$ and $\gamma^{\prime}$ intersects in some point $F_{2}$.

Since the first quadrant is positively invariant with respect to the flow associated to $(2.27)$ in $[\omega, \pi]$, we can find an interval $\left[\hat{s}_{k}, \check{s}_{k}\right] \subset\left(s_{k}, s_{k}^{\prime}\right)$ such that

$$
\begin{gathered}
z(\pi ; \omega, \gamma(s)) \in \mathcal{W}_{0}, \quad \forall s \in\left[\hat{s}_{k}, \check{s}_{k}\right] ; \\
\left|z\left(\pi ; \omega, \gamma\left(\hat{s}_{k}\right)\right)\right|=R_{1}, \quad\left|z\left(\pi ; \omega, \gamma\left(\check{s}_{k}\right)\right)\right|=R_{2} .
\end{gathered}
$$

It is now clear that the sub-path $\sigma_{(0,0)}^{k}=\left.\sigma\right|_{\left[\hat{s}_{k}, \check{s}_{k}\right]}$ satisfies the requirements of the assumption in Theorem 3.1.

Let us now fix $m \geq 1, \delta=\left(\delta_{1}, \ldots, \delta_{m}\right)$ and $k=\left(k_{1}, \ldots, k_{m}\right)$ as in the statement of Theorem 2.1. We show the existence of the solution $u_{k, \delta}$; the existence of the second solution $v_{k, \delta}$ can be obtained in a similar way.

For every $j=1, \ldots, m$, let us choose

$$
\delta_{j}^{\prime}=\left\{\begin{array}{l}
0 \text { if } k_{1}+\ldots+k_{j}+\delta_{1}+\ldots+\delta_{j} \text { is even } \\
1 \text { if } k_{1}+\ldots+k_{j}+\delta_{1}+\ldots+\delta_{j} \text { is odd }
\end{array}\right.
$$

and $\kappa=k$. We observe that $\delta_{m}=0$. We now apply Statement 1 of Theorem 3.1. It is easy to see that $u_{k, \delta}(\cdot)=x\left(\cdot ; 0, \tilde{p}_{\kappa, \delta^{\prime}}\right)$ is the solution to (2.1) we are looking for. Indeed, fixed points of $\psi^{m}$ are initial values of $m \pi$-periodic solutions to (2.1) and $u_{k, \delta}(0)>0$ and $\dot{u}_{k, \delta}(0)>0$ because $\tilde{p}_{\kappa, \delta^{\prime}} \in \mathcal{W}_{0}$.

Finally, $u_{k, \delta}$ has the required nodal properties by the previous discussion on $\eta$ and $\Lambda^{k}$ and the choice of $\delta^{\prime}$.

This concludes the proof of Statement 1. It is now clear how to obtain Statement 2 using the corresponding statement in Theorem 3.1. We have just to explain the boundedness of the solutions. A uniform bound of the solutions in the intervals of positivity of $q$ is easily deduced by assumption (2.13), Proposition 4.5, and Statement 2 of Proposition 4.2. Finally, the same bound can be extended in the intervals of negativity of $q$ by the argument employed in [17, Example 3].

Acknowledgements. The authors thank Professors F. Zanolin and S. Terracini for useful remarks on the subject of this paper.

\section{References}

1. Antonacci, F.: Existence of periodic solutions of Hamiltonian systems with potential indefinite in sign. Nonlinear Anal. 29, 1353-1364 (1997), DOI 10.1016/s0362546X(96)00190-3

2. Ávila, A., Felmer, P.L.: Periodic and subharmonic solutions for a class of second order Hamiltonian systems. Dyn. Syst. Appl. 3, 519-536 (1994)

3. Butler, G.J.: Rapid oscillation, nonextendability and the existence of periodic solutions to second order nonlinear differential equations. J. Differ. Equations 22, 467-477 (1976) 
4. Butler, G.J.: Periodic solutions of sublinear second order differential equations, J. Math. Anal. Appl. 62, 676-690 (1978)

5. Capietto, A., Dambrosio, W., Papini, D.: Superlinear indefinite equations on the real line and chaotic dynamics. J. Differ. Equations 181, 419-438 (2002), DOI 10.1006/jdeq.2001.4080

6. Ding, Y., Girardi, M.: Periodic and homoclinic solutions to a class of Hamiltonian systems with the potentials changing sign. Dyn. Syst. Appl. 2, 131-145 (1993)

7. Fabry, C., Habets, P.: Periodic solutions of second order differential equations with superlinear asymmetric nonlinearities. Arch. Math. (Basel) 60, 266-276 (1993)

8. Girardi, M., Matzeu, M.: Existence and multiplicity results for periodic solutions of superquadratic hamiltonian systems where the potential changes sign. NoDEA, Nonlinear Differ. Equ. Appl. 2, 35-61 (1995)

9. Jiang, M.-Y.: Subharmonic solutions of second order subquadratic Hamiltonian systems with potential changing sign, J. Math. Anal. Appl. 244, 291-303 (2000), DOI 10.1006/jmaa.1999.6687

10. Kirchgraber, U., Stoffer, D.: On the definition of chaos. ZAMM, Z. Angew. Math. Mech. 69, 175-185 (1989)

11. Lassoued, L.: Periodic solutions of a second order superquadratic system with a change of sign in the potential. J. Differ. Equations 93, 1-18 (1991)

12. Liu, B., Zanolin, F.: Subharmonic solutions of sublinear differential equations with indefinite weight. Preprint

13. Margheri, A., Rebelo, C., Zanolin, F.: Maslov index, Poincaré-Birkhoff theorem and periodic solutions of asymptotically linear planar Hamiltonian systems. J. Differ. Equations 183, 342-367 (2002), DOI 10.1006/jdeq.2001.4122

14. Mawhin, J., Papini, D., Zanolin, F.: Boundary blow-up for differential equations with indefinite weight, J. Differ. Equations 188, 33-51 (2003), DOI 10.1016/s00220396(02)00073-6

15. Papini, D.: Prescribing the nodal behaviour of periodic solutions of a superlinear equation with indefinite weight. Atti Sem. Mat. Fis. Univ. Modena 51, 43-63 (2003)

16. Papini, D., Zanolin, F.: A topological approach to superlinear indefinite boundary value problem. Topol. Methods Nonlinear Anal. 15, 203-233 (2000)

17. Papini, D., Zanolin, F.: Periodic points and chaotic-like dynamics of planar maps associated to nonlinear Hill's equations with indefinite weight. Georgian Math. J. 9, 339-366 (2002)

18. Schmitt, K.,Wang, Z.Q.: On critical points for noncoercive functionals and subharmonic solutions of some Hamiltonian systems. Electron. J. Differ. Equations Conf. 5, 237-245 (2000)

19. Terracini, S., Verzini, G.: Oscillating solutions to second-order ODEs with indefinite superlinear nonlinearities. Nonlinearity 13, 1501-1514 (2000), DOI 10.1088/0951$7715 / 13 / 5 / 305$ 